\title{
Stereoselective Synthesis of trans-2-Ethynyl-3-hydroxytetrahydropyran Derivatives
}

Eleuterio Alvarez, Ricardo Pérez, Milagros Rico, Rosa $\mathrm{M}^{\mathrm{a}}$ Rodríguez, $\mathrm{M}^{\mathrm{a}}$ Candelaria Suárez, and Julio D. Martín* Instituto Universitario de Bio-Orgánica “Antonio González”, Universidad de La Laguna-CSIC, Carretera Vieja de La Esperanza, 2; 38206 La Laguna, Tenerife, Spain

Fax: +34-(22)-630099

Received 12 August 1996

Abstract: Trans-substituted tetrahydropyrans with an acetylenic moiety at C-2 were synthesized from 2-formyloxy-3-(tertbutyldimethylsilyloxy)-tetrahydropyran by reaction with substituted silylacetylenes in the presence of a Lewis acid.

In the course of our studies towards the convergent synthesis of ciguatoxin and related compounds, ${ }^{1}$ we required a simple method for the construction of $\beta$-C-glycosyl derivatives. Stereoselective reduction of $\mathrm{C}-2$ hemiketals has been extensively investigated for alkynyl substituents, affording excellent yields of $\beta$-C-glycosides. ${ }^{2}$ A procedure involving $\alpha$-selective introduction of acetylenic groups to glucals followed by epimerization through dicobalt hexacarbonyl complex has also been reported recently. ${ }^{3,4}$

We wish to disclose in this letter our results dealing mainly with the synthesis of trans-2-ethynyl-3-hydroxytetrahydropyran derivatives, 3, by $\mathrm{C}_{2}$-alkynylation of $\mathbf{1}$ with substituted silylacetylenes in the presence of a Lewis acid (Scheme 1). The synthesis of $1\left(R_{1}=\right.$ TBS and $R_{1}=$ $\mathrm{Bn}$ ) is briefly summarized in Scheme 2. Diol $\mathbf{5}\left(\mathrm{R}_{1}=\mathrm{H}\right)^{5}$ was converted into $5\left(R_{1}=\right.$ TBS $)$ by silylation of both hydroxy groups with excess of $\mathrm{TBSCl}$ in DMF and selective deprotection at the primary position by removal of the TBS group with 0.2 equiv of $\mathrm{CSA}$ in $\mathrm{MeOH}$ at $0{ }^{\circ} \mathrm{C}$. Oxidation of $5\left(\mathrm{R}_{1}=\mathrm{TBS}\right)$ under Swern conditions gave aldehyde 6 which was further treated with $m$-CPBA to yield $1\left(\mathrm{R}_{1}=\mathrm{TBS}\right)(65 \%$ overall yield).<smiles>O=C1OCCC[C@H]1O</smiles>

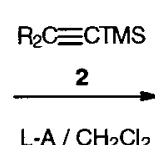

$\mathrm{L} \cdot \mathrm{A} / \mathrm{CH}_{2} \mathrm{Cl}_{2}$<smiles>[R]C#C[C@]1(O)CCCO[C@@H]1C</smiles><smiles>[2H]C#C[C@H]1OCC[C@@H](O)[C@@H]1O</smiles>

Scheme 1<smiles>CCO[C@H]1CCCO[C@@H]1CO</smiles><smiles></smiles>

5<smiles>[3H][IH]</smiles><smiles></smiles><smiles>C1CCCCC1</smiles><smiles>OC[C@H]1OCCC[C@@H]1O</smiles>

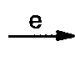<smiles>COC1(O)CCC[C@@H](O)[C@@H]1O</smiles>

$$
1
$$$$
\text { ib }
$$

Reagents and conditions: (a) From 5, $\mathbf{R}_{1}=$ TBS: 4.0 equiv of $\mathrm{SO}_{3}$.pyridine, 7.0 equiv of $\mathrm{Et}_{3} \mathrm{~N}, 6: 1 \mathrm{CH}_{2} \mathrm{Cl}_{2}$ :DMSO, $25^{\circ} \mathrm{C}, 4 \mathrm{~h}, 84 \%$; (b) 4.0 equiv of $m$-CPBA, $\mathrm{CH}_{2} \mathrm{Cl}_{2}, 0^{\circ} \mathrm{C}, 3 \mathrm{~h}, 77 \%$; (c) from $5, \mathbf{R}_{\mathbf{1}}=\mathbf{H}$ : 1.5 equiv of $\mathrm{PhCH}(\mathrm{OMe})_{2}, 0.01$ equiv of $\mathrm{CSA}, \mathrm{DMF}, 50^{\circ} \mathrm{C}, 4 \mathrm{~h}, 92 \%$; (d) 10.0 equiv of DIBAL, $\mathrm{CH}_{2} \mathrm{Cl}_{2}, 0^{\circ} \mathrm{C}, 30 \mathrm{~h}, 94 \%$; (e) 3.0 equiv of oxalyl chloride, 9.0 equiv of DMSO, 15.0 equiv of $\mathrm{Et}_{3} \mathrm{~N}, \mathrm{CH}_{2} \mathrm{Cl}_{2},-78$ ${ }^{\circ} \mathrm{C}, 30 \mathrm{~min}, 77 \%$.

Scheme 2

For the synthesis of $1\left(R_{1}=B n\right)$, diol $5\left(R_{1}=H\right)$ was selectively protected, by means of 1,3-benzylidene ketalization followed by DIBAL reduction to give 8 . Oxidation of 8 under Swern conditions gave aldehyde 9 which was further oxidized to formate $1\left(R_{1}=B n\right)$ by treatment with $m$-CPBA [51\% overall yield from $5\left(\mathrm{R}_{1}=\mathrm{H}\right)$ ].
Table 1

\begin{tabular}{|c|c|c|c|c|c|}
\hline Entry & Conditions $^{\mathrm{a}}$ & Substrate & Reagent & $\begin{array}{l}\text { Products } \\
\text { (Ratio) }^{\mathrm{b}}\end{array}$ & $\begin{array}{l}\text { Yield } \\
(\%)^{c}\end{array}$ \\
\hline 1 & A & $1, R_{1}=\mathbf{T B S}$ & $2, \mathbf{R}_{2}=\mathbf{H}$ & $\begin{array}{l}\mathbf{3 a}: \mathbf{4 a} \\
(0: 100)\end{array}$ & 60 \\
\hline 2 & B & $\mathbf{1}, \mathbf{R}_{1}=\mathbf{T B S}$ & $2, \mathbf{R}_{2}=\mathbf{H}$ & $\begin{array}{l}3 \mathbf{3}: \mathbf{4 a} \\
(0: 100)\end{array}$ & 73 \\
\hline 3 & A & $1, R_{1}=\mathbf{T B S}$ & $2, \mathbf{R}_{2}=\mathbf{T M S}$ & $\begin{array}{l}\mathbf{3 b}: \mathbf{4 b} \\
(66: 34)\end{array}$ & 74 \\
\hline 4 & B & $1, R_{1}=\mathbf{T B S}$ & $2, \mathbf{R}_{2}=\mathrm{TMS}$ & $\begin{array}{l}\mathbf{3 b}: \mathbf{4 b} \\
(80: 20)\end{array}$ & 68 \\
\hline 5 & A & $1, R_{1}=$ TBS & $2, \mathrm{R}_{2}=\mathrm{Me}$ & $\begin{array}{l}3 \mathbf{c}: \mathbf{4 c} \\
(70: 30)\end{array}$ & 81 \\
\hline 6 & B & $1, R_{1}=\mathbf{T B S}$ & $2, \mathbf{R}_{2}=\mathbf{M e}$ & $\begin{array}{l}3 c: 4 c \\
(100: 0)\end{array}$ & 67 \\
\hline 7 & A & $1, R_{1}=T B S$ & $2, \mathbf{R}_{2}=\mathbf{C}_{3} \mathbf{H}_{7}$ & $\begin{array}{l}3 \mathbf{3 d}: \mathbf{4 d} \\
(80: 20)\end{array}$ & 66 \\
\hline 8 & B & $1, R_{1}=$ TBS & $2, \mathbf{R}_{2}=\mathbf{C}_{3} \mathbf{H}_{7}$ & $\begin{array}{l}3 \mathbf{3 d}: \mathbf{4 d} \\
(90: 10)\end{array}$ & 72 \\
\hline 9 & A & $1, R_{1}=\mathrm{TBS}$ & $2, \mathbf{R}_{2}=\mathbf{C}_{6} \mathbf{H}_{5}$ & $\begin{array}{l}\mathbf{3 e}: \mathbf{4 e} \\
(80: 20)\end{array}$ & 64 \\
\hline 10 & B & $\mathbf{1}, \mathrm{R}_{1}=\mathbf{T B S}$ & $2, \mathbf{R}_{2}=\mathbf{C}_{6} \mathbf{H}_{5}$ & $\begin{array}{l}3 \mathbf{e}: \mathbf{4 e} \\
(95: 5)\end{array}$ & 71 \\
\hline
\end{tabular}

${ }^{a}$ Method A: 1.0 equiv $\mathrm{TiCl}_{4}, \mathrm{CH}_{2} \mathrm{Cl}_{2},-78^{\circ} \mathrm{C}$ to $-20^{\circ} \mathrm{C}$; Method B: 1.0 equiv $\mathrm{SnCl}_{4}, \mathrm{CH}_{2} \mathrm{Cl}_{2},-78{ }^{\circ} \mathrm{C}$ to $-20^{\circ} \mathrm{C}$. ${ }^{\mathrm{b}} \mathrm{Compounds} 3$ and 4 could be separated by silica gel chromatography in pure form. Ratio was determined on the basis of the isolated amount of each compound, 3 and 4 . $^{\mathrm{c}}$ The specific yields are isolated yields of $\mathbf{3}$ and 4 .

While the two differently substituted substrates $1\left(\mathrm{R}_{1}=\right.$ TBS and $\left.\mathrm{Bn}\right)$ were tried out with a variety of Lewis acids (e.g. TMSOTf, $\mathrm{TiCl}_{4}$, $\mathrm{SnCl}_{4}, \mathrm{BF}_{3} \cdot \mathrm{Et}_{2} \mathrm{O}$ and $\mathrm{ZnBr}_{2}$ ), solvents, reaction temperatures and times, a very simple procedure which called for the dropwise addition of $\mathrm{TiCl}_{4}$ (or $\mathrm{SnCl}_{4}$ ) solution (1.0 equiv, $1 \mathrm{M}$ in $\mathrm{CH}_{2} \mathrm{Cl}_{2}$ ) to a cooled ($\left.78{ }^{\circ} \mathrm{C}\right)$ mixture of $1\left(\mathrm{R}_{1}=\mathrm{TBS}\right)^{6}$ and substituted silylacetylene $(2.0$ equiv) in $\mathrm{CH}_{2} \mathrm{Cl}_{2}$ proved to be the most satisfactory. The reaction mixture was allowed to warm up to $-20^{\circ} \mathrm{C}$ and was stirred for $15-30$ min. Work-up and chromatography afforded $\mathrm{C}_{2}$-alkynylated products (Table 1). The stereochemistry assigned to compounds 3a-e and 4a-e was supported by the coupling constants $\left(\mathrm{J}_{\mathrm{a}, \mathrm{b}}\right)$ between $\mathrm{C}-2$ and $\mathrm{C}-3$ protons determined by analysis of the ${ }^{1} \mathrm{H}$ NMR spectra and set out in Table 2.,10

Table 2. ${ }^{1} \mathrm{H}$ NMR Data Coupling Constants
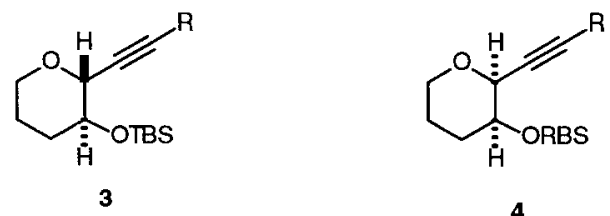

\begin{tabular}{cccc}
\hline Compound & $\mathrm{J}_{\mathrm{a}, \mathrm{b}}(\mathrm{Hz})$ & Compound & $\mathbf{J}_{\mathbf{a}, \mathrm{b}}(\mathrm{Hz})$ \\
\hline 3a $(\mathbf{R}=\mathbf{H})$ & $7.7^{7}$ & $\mathbf{4 a}(\mathbf{R}=\mathbf{H})$ & 4.2 \\
3b $(\mathbf{R}=\mathbf{T M S})$ & 8.2 & $\mathbf{4 b}(\mathbf{R}=\mathbf{T M S})$ & 4.2 \\
3c $(\mathbf{R}=\mathbf{M e})$ & 8.0 & $\mathbf{4 c}(\mathbf{R}=\mathbf{M e})$ & 4.5 \\
$\mathbf{3 d}\left(\mathbf{R}=\mathbf{C}_{\mathbf{3}} \mathbf{H}_{7}\right)$ & 8.8 & $\mathbf{4 d}\left(\mathbf{R}=\mathbf{C}_{\mathbf{3}} \mathbf{H}_{7}\right)$ & 4.2 \\
$\mathbf{3 e}\left(\mathbf{R}=\mathbf{C}_{\mathbf{6}} \mathbf{H}_{5}\right)$ & 7.9 & $\mathbf{4 e}\left(\mathbf{R}=\mathbf{C}_{\mathbf{6}} \mathbf{H}_{\mathbf{5}}\right)$ & 4.3 \\
\hline
\end{tabular}

With regard to the stereochemistry of this alkynylation process, Table 1 clearly shows that when terminal substituted silylacetylenes $2\left(R_{2} \neq\right.$ $\mathrm{H})$ were used, the reaction appeared superficially to take on more of 
Compound $^{a}$<smiles>O=CO[C@H]1OCCC[C@H]1O</smiles>

1<smiles>CC(=O)O[C@H]1O[C@H](COCc2ccccc2)[C@@H](Cc2ccccc2)[C@H](OCc2ccccc2)[C@H]1Cc1ccccc1</smiles>

12 (ref 8 )

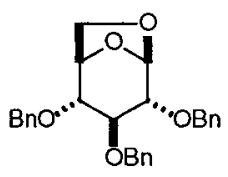

15 (ref 9) Products cis:trans (ratio)

Yield (\%)<smiles>C=CC[C@H]1OCCC[C@H]1O[SbH3]</smiles><smiles>C=CC[C@H]1OCCC[C@H]1O[Na]</smiles>

10:11 (1:5)<smiles>C=CC[C@H]1O[C@H](COc2ccccc2)[C@@H](Cc2ccccc2)[C@@H](O)[C@]1(O)c1ccccc1</smiles><smiles>C=CC[C@H]1O[C@H](COCc2ccccc2)[C@@H](Cc2ccccc2)[C@@H](O)[C@]1(O)c1ccccc1</smiles>

13:14 $(\geq 10: 1)$

14

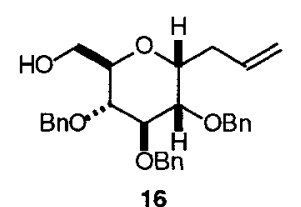

16:17 ( $\geq 10: 1)$

55

Reaction conditions: Dropwise addition of TMSOTf soln (1.0 equiv, $1 \mathrm{M}$ in $\left.\mathrm{CH}_{2} \mathrm{Cl}_{2}\right)$ to a cooled $\left(-78^{\circ} \mathrm{C}\right)$ mixture of activated pyranoside and allyltrimethylsilane ( 1.5 equiv) in $\mathrm{CH}_{2} \mathrm{Cl}_{2}$. The reaction mixture was allowed to warm to $-20^{\circ} \mathrm{C}$ and stirred for $30 \mathrm{~min}$.

the character of an $S_{N} 2$ process (entries $3-10$ ). In these cases, an efficient blocking of one face of the oxane ring by the TBSO group forced the silylacetylenes to approach from the back giving preferentially trans-substituted compounds. The trimethylsilyl acetylene itself $\left(2, R_{2}=H\right)$, on the other hand, showed a preference for forming a product in which the $\mathrm{C}-2$ stereochemistry was retained (entries 1 and 2, Table 1).

Along this line, it is worth adding that the treatment of $1\left(R_{1}=T B S\right)$ with allyltrimethylsilane gave a good yield $(93 \%)$ of $\mathrm{C}_{2}$-allylated products, cis:trans (1:5) ratio, under reaction conditions in which activated glucopyranosides are stereoselective to $\alpha$-allylation (Table 3).

Acknowledgement. Financial support from the Ministry of Education and Science, Spain (PB92-0487) and the EU (Contract CI1-CT920049) is gratefully acknowledged.

\section{References and Notes}

(1) For previous studies from our group, see: Alvarez, E.; Pérez, R.; Rodríguez, R.M.; Martín, J.D. J. Org. Chem. 1996, 61, 3003.

(2) (a) Lewis, M.D.; Cha, J.K.; Kishi, Y. J. Am. Chem. Soc. 1982, 104, 4976. (b) Rouzand, D.; Sinay, P. J.C.S., Chem. Commun. 1983, 1353. (c) Giannis, A.; Sandhoff, K. Tetrahedron Lett. 1985, 26, 1479. (d) Daly, S.M.; Armstrong, R.W. Tetrahedron Letts. 1989, 30, 5713. (e) Sutherlin, D.P.; Armstrong, R.W. Tetrahedron Lett. 1993, 34, 4897.

(3) (a) Ichikawa, Y.; Isobe, M.; Konobe, M.; Goto, T. Carbohydrate Research 1987, 171, 193, (b) Isobe, M.; Tsukiyama, Y. Tetrahedron Lett. 1992, 33, 7911.(c) Tsukiyama, T.; Peters, S.C.; Isobe, M. Synlett. 1993, 413. (d) Hosokawa, S.; Isobe, M. Synlett. 1995, 1179.

(4) (a)Tanaka, S.; Tsukiyama, T.; Isobe, M. Tetrahedron Lett. 1993, 34, 5757. (b) Tanaka, S.; Isobe, M. Tetrahedron 1994, $50,5633$.

(5) Nicolaou, K.C.; Hwang, C.-K.; Marron, B.E.; De Frees, S.A.; Couladouros, E.A.; Abe, Y.; Caroll, P.J.; Snyder, J.P. I. Am. Chem. Soc. 1990, 112, 3040.

(6) Formate ester $1\left(R_{1}=\right.$ TBS or $\left.B n\right)$ was chosen on the basis of the compatibility of this group with $\mathrm{TiCl}_{4}$ and $\mathrm{SnCl}_{4}$ which precluded hydrolysis of the ester during the reaction process.

(7) The trans-isomer 3a was synthesized via dibromoolefination of aldehyde 6 followed by treatment with 2.0 equiv of $n$-BuLi in THF: Corey, E.J.; Fuchs, P.L. Tetrahedron Lett. 1972, 3769.
(8) Compound 12 was prepared by acylation $\left(\mathrm{Ac}_{2} \mathrm{O} /\right.$ cat DMAP cat/TEA/CH $\mathrm{CH}_{2}$ ) of 2,3,4,6-tetrabenzylglucopyranose.

(9) 2,3,4-Tribenzyl-1,6-anhydro-D-glucopyranose (15) was prepared fron $\alpha-D(+)$ glucose pentaacetate in $64 \%$ overall yield as illustrated below:<smiles>CC(=O)OC[C@H]1O[C@H](OC(C)=O)[C@@H](OC(C)=O)[C@H](OC(C)=O)[C@@H]1OC(C)=O</smiles><smiles>CC(=O)OC[C@H]1O[C@@H](c2ccccc2)[C@H](OC(C)=O)[C@H](OC(C)=O)[C@@H]1OC(C)=O</smiles><smiles>CC(=O)OC[C@H]1O[C@@H](S(=O)(=O)c2ccccc2)[C@H](OC(C)=O)[C@H](OC(C)=O)[C@@H]1OC(C)=O</smiles>

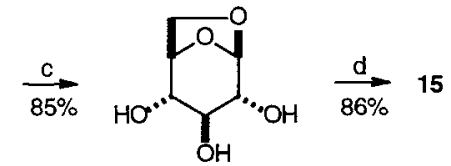

Reagents and conditions: (a) 1.5 equiv of PhSTMS, 1.2 equiv of TMSOTf, $\mathrm{CH}_{2} \mathrm{Cl}_{2}, 0-25{ }^{\circ} \mathrm{C}, 6 \mathrm{~h}, 95 \%$, [ca. $\alpha: \beta(5: 1)$ stereoselectivity]; (b) 3.0 equiv of $m$-CPBA, 3.0 equiv of $\mathrm{NaHCO}_{3}$, EtOAc, $0-25^{\circ} \mathrm{C}, 12 \mathrm{~h}, 92 \%$; (c) 3.0 of NaOMe, $\mathrm{MeOH}, 0-25^{\circ} \mathrm{C}, 2 \mathrm{~h}, 85 \%$; (d) 3.1 equiv of $\mathrm{NaH}, 3.1$ equiv of $\mathrm{BnBr}$, cat. $\mathrm{n}-\mathrm{Bu}_{4} \mathrm{NI}, \mathrm{THF}, 0^{\circ} \mathrm{C}, 12 \mathrm{~h}, 86 \%$.

For a comparative study with reported methods for synthesis of 1,6-anhydro-D-glucopyranose, see: Zottola, M.A.; Alonso, R.; Vite, G.D.; Fraser-Reid, B. J. Org. Chem. 1989, 54, 6123, and references quoted therein.

(10) All products gave spectroscopic data consistent with the proposed structures. For example, $1\left(\mathbf{R}_{1}=\right.$ TBS): oil; $[\alpha]_{\mathrm{D}}$ $+17.7^{\circ}\left(\mathrm{c} 0.94, \mathrm{CHCl}_{3}\right) ;{ }^{1} \mathrm{H}$ NMR $\left(400 \mathrm{MHz}, \mathrm{CDCl}_{3}\right): \delta 8.12$ $(\mathrm{s}, 1 \mathrm{H}), 5.63(\mathrm{~d}, 1 \mathrm{H}, \mathrm{J}=4.4 \mathrm{~Hz}), 3.89(\mathrm{ddd}, 1 \mathrm{H}, \mathrm{J}=11.4,7.7$, $3.3 \mathrm{~Hz}$ ), 3.66 (ddd, $1 \mathrm{H}, \mathrm{J}=11.4,3.6,3.2 \mathrm{~Hz}$ ), $3.62(\mathrm{ddd}, 1 \mathrm{H}, \mathrm{J}$ $=8.0,4.3,3.1 \mathrm{~Hz}), 1.96(\mathrm{~m}, 2 \mathrm{H}), 1.64(\mathrm{~m}, 1 \mathrm{H}), 1.50(\mathrm{~m}, 1 \mathrm{H})$, $0.91(\mathrm{~s}, 9 \mathrm{H}), 0.07(\mathrm{~s}, 6 \mathrm{H}) ;{ }^{13} \mathrm{C}$ NMR $\left(100 \mathrm{MHz}, \mathrm{CDCl}_{3}\right): \%$ 160.1 (d), 96.1 (d), 67.5 (d), 64.5 (t), 29.1 (t), 26.1 (q), 21.8 (t), 18.4 (s), $-4.4(\mathrm{q})$; HRMS Calcd for $\mathrm{C}_{11} \mathrm{H}_{23} \mathrm{O}_{2} \mathrm{Si}\left(\mathrm{M}^{+}-\right.$ $\left.\mathrm{CO}_{2} \mathrm{H}\right): 215.14673$; Found: $215.14336 .3\left(\mathbf{R}_{1}=\mathbf{T B S}, \mathbf{R}_{\mathbf{2}}=\mathbf{H}\right)$ : oil; $[\alpha]_{\mathrm{D}}+29.3^{\circ}\left(\mathrm{c} 1.2, \mathrm{CHCl}_{3}\right) ;{ }^{1} \mathrm{H} \mathrm{NMR}\left(400 \mathrm{MHz}, \mathrm{CDCl}_{3}\right): \delta$ 3.91 (dddd, $1 \mathrm{H}, \mathrm{J}=11.4,4.2,3.8,1.2 \mathrm{~Hz}$ ), 3.87 (dd, $1 \mathrm{H}, \mathrm{J}=$ $7.7,2.0 \mathrm{~Hz}$ ), 3.61 (ddd, $1 \mathrm{H}, \mathrm{J}=9.1,7.7,4.2 \mathrm{~Hz}$ ), 3.39 (ddd, $1 \mathrm{H}, \mathrm{J}=11.4,10.1,2.8 \mathrm{~Hz}), 2.42(\mathrm{~d}, 1 \mathrm{H}, \mathrm{J}=2.1 \mathrm{~Hz}), 2.04$ (ddddd, $1 \mathrm{H}, \mathrm{J}=13.1,5.1,4.2,3.8,1.1 \mathrm{~Hz}$ ), 1.72 (br ddd, $1 \mathrm{H}, \mathrm{J}$ $=11.8,4.2,3.8 \mathrm{~Hz}), 1.62(\mathrm{~m}, 1 \mathrm{H}), 1.45$ (dddd, $1 \mathrm{H}, \mathrm{J}=13.1$, $11.2,9.1,4.2 \mathrm{~Hz}), 0.89(\mathrm{~s}, 9 \mathrm{H}), 0.10(\mathrm{~s}, 3 \mathrm{H}), 0.08(\mathrm{~s}, 3 \mathrm{H}) ;{ }^{13} \mathrm{C}$ NMR (100 MHz, $\left.\mathrm{CDCl}_{3}\right): \delta 82.1$ (d), 73.7 (s), 72.8 (d), 70.3 
(d), $66.7(\mathrm{t}), 32.1(\mathrm{t}), 25.7(\mathrm{q}), 24.0(\mathrm{t}), 18.0(\mathrm{~s}),-4.5(\mathrm{q}),-4.6$ (q); HRMS Caled for $\mathrm{C}_{13} \mathrm{H}_{23} \mathrm{O}_{2} \mathrm{Si}\left(\mathrm{M}^{+}-\mathrm{H}\right)$ : 239.14673; Found: 239.14638. $4\left(\mathbf{R}_{1}=\right.$ TBS, $\left.\mathbf{R}_{2}=\mathbf{H}\right)$ : oil; $[\alpha]_{D}-79.1^{\circ}$ (c 0.46, $\left.\mathrm{CHCl}_{3}\right) ;{ }^{1} \mathrm{H} \mathrm{NMR}\left(400 \mathrm{MHz}, \mathrm{CDCl}_{3}\right): \delta 4.48(\mathrm{dd}, 1 \mathrm{H}, \mathrm{J}=4.2$, $1.7 \mathrm{~Hz}$ ), 3.86 (ddd, $1 \mathrm{H}, \mathrm{J}=11.4,10.7,2.6 \mathrm{~Hz}$ ), 3.76 (ddd, $1 \mathrm{H}$, $\mathrm{J}=9.0,4.7,4.2 \mathrm{~Hz}$ ), 3.59 (dddd, $1 \mathrm{H}, \mathrm{J}=11.5,4.3,3.4,0.9$ $\mathrm{Hz}), 2.44(\mathrm{~d}, 1 \mathrm{H}, \mathrm{J}=2.2 \mathrm{~Hz}), 1.78(\mathrm{~m}, 3 \mathrm{H}), 1.59(\mathrm{~m}, 1 \mathrm{H}), 0.90$ $(\mathrm{s}, 9 \mathrm{H}), 0.09(\mathrm{~s}, 3 \mathrm{H}), 0.08(\mathrm{~s}, 3 \mathrm{H}) ;{ }^{13} \mathrm{C}$ NMR $(100 \mathrm{MHz}$, $\left.\mathrm{CDCl}_{3}\right): \delta 80.3(\mathrm{~s}), 75.7(\mathrm{~s}), 70.8(\mathrm{~d}), 68.5(\mathrm{~d}), 63.2(\mathrm{t}), 29.5$ (t), $26.2(\mathrm{q}), 24.2(\mathrm{t}), 18.5(\mathrm{~s}),-4.20(\mathrm{q}),-4.30(\mathrm{q})$; HRMS Calcd for $\mathrm{C}_{13} \mathrm{H}_{23} \mathrm{O}_{2} \mathrm{Si}\left(\mathrm{M}^{+}-\mathrm{H}\right): 239.14673$; Found: 239.14663 .3 $\left(\mathbf{R}_{1}=\mathbf{T B S}, \mathbf{R}_{\mathbf{2}}=\mathbf{T M S}\right)$ : oil; $[\alpha]_{\mathrm{D}}+34.6^{\circ}\left(\mathrm{c} 0.43, \mathrm{CHCl}_{3}\right) ;{ }^{1} \mathrm{H}$ NMR $\left(400 \mathrm{MHz}, \mathrm{CDCl}_{3}\right): \delta 3.91$ (dddd, $1 \mathrm{H}, \mathrm{J}=11.4,3.1,2.8$, $1.4 \mathrm{~Hz}), 3.83(\mathrm{~d}, 1 \mathrm{H}, \mathrm{J}=8.2 \mathrm{~Hz}), 3.56(\mathrm{ddd}, 1 \mathrm{H}, \mathrm{J}=9.7,8.3$, $4.4 \mathrm{~Hz}$ ), 3.36 (ddd, $1 \mathrm{H}, \mathrm{J}=11.4,10.3,3.8 \mathrm{~Hz}), 2.05(\mathrm{~m}, 1 \mathrm{H})$, $1.63(\mathrm{~m}, 2 \mathrm{H}), 1.43(\mathrm{~m}, 1 \mathrm{H}), 0.90(\mathrm{~s}, 9 \mathrm{H}), 0.15(\mathrm{~s}, 9 \mathrm{H}), 0.12(\mathrm{~s}$, $3 \mathrm{H}), 0.10(\mathrm{~s}, 3 \mathrm{H}) ;{ }^{13} \mathrm{C} \mathrm{NMR}\left(100 \mathrm{MHz}, \mathrm{CDCl}_{3}\right): \delta 104.2(\mathrm{~s})$, $91.0(\mathrm{~s}), 74.1(\mathrm{~d}), 70.8(\mathrm{~d}), 67.6(\mathrm{t}), 33.1(\mathrm{t}), 26.2(\mathrm{q}), 24.8(\mathrm{t})$, $18.5(\mathrm{~s}), 0.2(\mathrm{q}),-4.0(\mathrm{q}),-4.1(\mathrm{q})$; HRMS Calcd for $\mathrm{C}_{16} \mathrm{H}_{32} \mathrm{O}_{2} \mathrm{Si}_{2}\left(\mathrm{M}^{+}\right):$312.19409; Found: 312.19396. $4\left(\mathbf{R}_{1}=\right.$ TBS, $\mathbf{R}_{\mathbf{2}}=$ TMS): oil; $[\alpha]_{\mathrm{D}}-14.4^{\circ}$ (c 1.35, $\left.\mathrm{CHCl}_{3}\right) ;{ }^{1} \mathrm{H}$ NMR $\left(400 \mathrm{MHz}, \mathrm{CDCl}_{3}\right): \delta 4.42(\mathrm{~d}, 1 \mathrm{H}, \mathrm{J}=4.2 \mathrm{~Hz}$ ), 3.87 (ddd, $1 \mathrm{H}$, $\mathbf{J}=11.6,9.1,2.7 \mathrm{~Hz}), 3.75$ (ddd, $1 \mathrm{H}, \mathrm{J}=8.0,4.2,3.8 \mathrm{~Hz}$ ), 3.57 (dddd, $1 \mathrm{H}, \mathrm{J}=11.4,5.0,4.7,1.1 \mathrm{~Hz}), 1.78(\mathrm{~m}, 3 \mathrm{H}), 1.49$ $(\mathrm{m}, 1 \mathrm{H}), 0.90(\mathrm{~s}, 9 \mathrm{H}), 0.17(\mathrm{~s}, 9 \mathrm{H}), 0.09(\mathrm{~s}, 3 \mathrm{H}), 0.08(\mathrm{~s}, 3 \mathrm{H})$; ${ }^{13} \mathrm{C} \mathrm{NMR}\left(100 \mathrm{MHz}, \mathrm{CDCl}_{3}\right.$ ): $\delta 102.4$ (s), 92.3 (s), 71.5 (d), $68.4(\mathrm{~d}), 64.1(\mathrm{t}), 29.9(\mathrm{t}), 26.1(\mathrm{q}), 23.6(\mathrm{q}), 18.4(\mathrm{~s}), 0.3(\mathrm{q})$, 4.2; (q); HRMS Calcd for $\mathrm{C}_{11} \mathrm{H}_{23} \mathrm{O}_{2} \mathrm{Si}\left(\mathrm{M}^{+}-{ }^{-} \mathrm{Bu}\right)$ : 255.21442; Found: $255.21454 ; 3\left(\mathbf{R}_{1}=\right.$ TBS, $\left.\mathbf{R}_{\mathbf{2}}=\mathbf{C H}_{3}\right)$ : oil; $[\alpha]_{\mathrm{D}}+31.4^{\circ}$ (c 1.0, $\mathrm{CHCl}_{3}$ ); ${ }^{1} \mathrm{H} \mathrm{NMR}\left(400 \mathrm{MHz}, \mathrm{CDCl}_{3}\right.$ ): $\delta 3.89$ (dddd, $1 \mathrm{H}$, $\mathrm{J}=11.4,4.3,2.9,1.4 \mathrm{~Hz}), 3.81(\mathrm{dd}, 1 \mathrm{H}, \mathrm{J}=8.0,2.0 \mathrm{~Hz}), 3.55$ (ddd, $1 \mathrm{H}, \mathrm{J}=9.5,8.0,4.3 \mathrm{~Hz}$ ), 3.36 (ddd, $1 \mathrm{H}, \mathrm{J}=11.4,10.6$, $3.1 \mathrm{~Hz}), 2.02(\mathrm{~m}, 1 \mathrm{H}), 1.84(\mathrm{~d}, 3 \mathrm{H}, \mathrm{J}=2.1 \mathrm{~Hz}), 1.62(\mathrm{~m}, 2 \mathrm{H})$, $1.44(\mathrm{~m}, 1 \mathrm{H}), 0.90(\mathrm{~s}, 9 \mathrm{H}), 0.11(\mathrm{~s}, 3 \mathrm{H}), 0.09(\mathrm{~s}, 3 \mathrm{H}) ;{ }^{13} \mathrm{C}$ NMR $\left(100 \mathrm{MHz}, \mathrm{CDCl}_{3}\right): \delta 82.1(\mathrm{~s}), 78.1(\mathrm{~s}), 73.8$ (d), 71.3 (d), $67.3(\mathrm{t}), 32.9(\mathrm{t}), 26.1(\mathrm{q}), 24.9(\mathrm{t}), 18.5(\mathrm{~s}), 4.0(\mathrm{q}),-4.2$ (q), - 4.3 (q); HRMS Calcd for $\mathrm{C}_{14} \mathrm{H}_{25} \mathrm{O}_{2} \mathrm{Si}\left(\mathrm{M}^{+}-\mathrm{H}\right): 253.16238$; Found: $253.16336 .4\left(\mathbf{R}_{\mathbf{1}}=\mathbf{T B S}, \mathbf{R}_{\mathbf{2}}=\mathbf{C H}_{\mathbf{3}}\right)$ : oil; $[\alpha]_{\mathrm{D}}-77.7^{\circ}(\mathrm{c}$ $\left.0.53, \mathrm{CHCl}_{3}\right) ;{ }^{1} \mathrm{H} \mathrm{NMR}\left(400 \mathrm{MHz}, \mathrm{CDCl}_{3}\right): \delta 4.38(\mathrm{dd}, 1 \mathrm{H}, \mathrm{J}=$ $4.5,2.0 \mathrm{~Hz}$ ), 3.87 (ddd, $1 \mathrm{H}, \mathrm{J}=11.5,9.1,2.7 \mathrm{~Hz}$ ), 3.74 (ddd, $1 \mathrm{H}, \mathrm{J}=8.1,4.2,3.9 \mathrm{~Hz}$ ), 3.54 (dddd, $1 \mathrm{H}, \mathrm{J}=11.4,4.8,3.9$, $0.9 \mathrm{~Hz}), 1.87(\mathrm{~d}, 3 \mathrm{H}, \mathrm{J}=2.2 \mathrm{~Hz}), 1.76(\mathrm{~m}, 3 \mathrm{H}), 1.50(\mathrm{~m}, 1 \mathrm{H})$, $0.90(\mathrm{~s}, 9 \mathrm{H}), 0.09(\mathrm{~s}, 3 \mathrm{H}), 0.07(\mathrm{~s}, 3 \mathrm{H}) ;{ }^{13} \mathrm{C}$ NMR $(100 \mathrm{MHz}$, $\left.\mathrm{CDCl}_{3}\right): \delta 83.5(\mathrm{~s}), 75.9(\mathrm{~s}), 71.3(\mathrm{~d}), 68.9(\mathrm{~d}), 63.9(\mathrm{t}), 29.9$ $(\mathrm{t}), 26.2(\mathrm{q}), 23.7(\mathrm{t}), 18.6(\mathrm{~s}),-4.0(\mathrm{q}),-4.3(\mathrm{q})$; HRMS Calcd for $\mathrm{C}_{14} \mathrm{H}_{25} \mathrm{O}_{2} \mathrm{Si}\left(\mathrm{M}^{+}-\mathrm{H}\right): 253.16238$; Found: $253.15980 .3\left(\mathbf{R}_{1}=\right.$ TBS, $\left.\mathbf{R}_{2}=\mathbf{C}_{\mathbf{3}} \mathbf{H}_{7}\right)$ : oil; $[\alpha]_{\mathrm{D}}+58^{\circ}\left(\mathrm{c} 0.17, \mathrm{CHCl}_{3}\right) ;{ }^{1} \mathrm{H}$ NMR $\left(400 \mathrm{MHz}, \mathrm{CDCl}_{3}\right): \delta 3.90$ (dddd, $1 \mathrm{H}, \mathrm{J}=11.4,4.4,4.2,1.1$ $\mathrm{Hz}), 3.86$ (ddd, $1 \mathrm{H}, \mathrm{J}=8.8,1.8,1.8 \mathrm{~Hz}$ ), 3.54 (ddd, $1 \mathrm{H}, \mathrm{J}=$ $9.3,7.9,4.3 \mathrm{~Hz}$ ), 3.38 (ddd, $1 \mathrm{H}, \mathrm{J}=11.3,10.5,3.0 \mathrm{~Hz}$ ), 2.20 (ddd, $2 \mathrm{H}, \mathrm{J}=7.2,7.1,2.0 \mathrm{~Hz}$ ), 2.00 (dddd, $1 \mathrm{H}, \mathrm{J}=12.6,7.5$, $4.0,3.5 \mathrm{~Hz}), 1.66(\mathrm{~m}, 1 \mathrm{H}), 1.61(\mathrm{~m}, 1 \mathrm{H}), 1.53(\mathrm{~m}, 2 \mathrm{H}), 1.43$ $(\mathrm{m}, 1 \mathrm{H}), 0.98(\mathrm{dd}, 3 \mathrm{H}, \mathrm{J}=7.4,7.4 \mathrm{~Hz}), 0.90(\mathrm{br} \mathrm{s}, 9 \mathrm{H}), 0.11$ (s, 3H), $0.09(\mathrm{~s}, 3 \mathrm{H}) ;{ }^{13} \mathrm{C}$ NMR $\left(100 \mathrm{MHz}, \mathrm{CDCl}_{3}\right): \delta 86.7(\mathrm{~s})$ $78.4(\mathrm{~s}), 73.4(\mathrm{~d}), 70.8(\mathrm{~d}), 66.8(\mathrm{t}), 32.4(\mathrm{t}), 25.8(\mathrm{q}), 24.3(\mathrm{t})$, $21.9(\mathrm{t}), 20.8(\mathrm{t}), 13.6(\mathrm{~s}),-4.5(\mathrm{q}),-4.6(\mathrm{q})$; HRMS Calcd for $\mathrm{C}_{16} \mathrm{H}_{30} \mathrm{O}_{2} \mathrm{Si}\left(\mathrm{M}^{+}\right)$: 282.20151; Found: $282.20174 .4\left(\mathbf{R}_{1}=\right.$ TBS, $\left.\mathbf{R}_{2}=\mathbf{C}_{3} \mathbf{H}_{7}\right):$ oil; $[\alpha]_{\mathrm{D}}-52^{\circ}$ (c $\left.0.10, \mathrm{CHCl}_{3}\right) ;{ }^{1} \mathrm{H}$ NMR $(400$ $\left.\mathrm{MHz}, \mathrm{CDCl}_{3}\right): \delta 4.45(\mathrm{dd}, 1 \mathrm{H}, \mathrm{J}=4.2,1.8 \mathrm{~Hz}$ ), $3.87(\mathrm{ddd}, 1 \mathrm{H}$, $\mathrm{J}=11.5,10.0,2.8 \mathrm{~Hz}$ ), $3.72(\mathrm{ddd}, 1 \mathrm{H}, \mathrm{J}=8.8,4.2,4.0 \mathrm{~Hz}$ ), $3.55(\mathrm{ddd}, 1 \mathrm{H}, \mathrm{J}=11.5,5.0,3.8 \mathrm{~Hz}), 2.21(\mathrm{ddd}, 2 \mathrm{H}, \mathrm{J}=7.0$, $7.0,2.0 \mathrm{~Hz}), 1.56(\mathrm{~m}, 4 \mathrm{H}), 1.54(\mathrm{~m}, 2 \mathrm{H}), 1.00(\mathrm{dd}, 3 \mathrm{H}, \mathrm{J}=7.4$, $7.3 \mathrm{~Hz}), 0.90(\mathrm{~s}, 9 \mathrm{H}), 0.08(\mathrm{~s}, 3 \mathrm{H}), 0.00(\mathrm{~s}, 3 \mathrm{H}) ;{ }^{13} \mathrm{C} \mathrm{NMR}$ $\left(100 \mathrm{MHz}, \mathrm{CDCl}_{3}\right): \delta 87.8$ (s) 70.8 (d), $68.5(\mathrm{~d}), 63.0(\mathrm{t}), 29.3$ $(\mathrm{t}), 25.7(\mathrm{q}), 23.6(\mathrm{t}), 22.1(\mathrm{t}), 20.8(\mathrm{t}), 18.1(\mathrm{~s}), 13.5(\mathrm{q}),-4.6$ (q); HRMS Calcd for $\mathrm{C}_{16} \mathrm{H}_{30} \mathrm{O}_{2} \mathrm{Si}\left(\mathrm{M}^{+}\right)$: 282.20151; Found: $282.19677 .3\left(\mathbf{R}_{1}=\mathbf{T B S}, \mathbf{R}_{\mathbf{2}}=\mathbf{C}_{6} \mathbf{H}_{5}\right)$ : oil; $[\alpha]_{\mathrm{D}}+38.1^{\circ}$ (c 0.53 , $\left.\mathrm{CHCl}_{3}\right) ;{ }^{1} \mathrm{H}$ NMR $\left(400 \mathrm{MHz}, \mathrm{CDCl}_{3}\right): \delta 7.45(\mathrm{~m}, 2 \mathrm{H}), 7.29(\mathrm{~m}$, $3 \mathrm{H}), 4.09(\mathrm{~d}, 1 \mathrm{H}, \mathrm{J}=7.9 \mathrm{~Hz}$ ), 3.98 (ddd, $1 \mathrm{H}, \mathrm{J}=11.4,3.3,3.1$ $\mathrm{Hz}$ ), 3.69 (ddd, $1 \mathrm{H}, \mathrm{J}=9.2,7.9,4.3 \mathrm{~Hz}$ ), $3.45(\mathrm{ddd}, 1 \mathrm{H}, \mathrm{J}=$ $11.2,10.5,3.2 \mathrm{~Hz}$ ), 2.09 (dddd, $1 \mathrm{H}, \mathrm{J}=8.0,7.7,4.0,3.6 \mathrm{~Hz}$ ), $1.74(\mathrm{~m}, 1 \mathrm{H}), 1.68(\mathrm{~m}, 1 \mathrm{H}), 1.50(\mathrm{~m}, 1 \mathrm{H}), 0.92(\mathrm{br} \mathrm{s}, 9 \mathrm{H}), 0.13$ (s, 6H); ${ }^{13} \mathrm{C}$ NMR (100 MHz, $\left.\mathrm{CDCl}_{3}\right): \delta 131.7$ (d), 128.2 (d), 128.1 (d), $122.8(\mathrm{~s}), 87.5(\mathrm{~s}), 85.5(\mathrm{~s}), 73.7$ (d), $70.6(\mathrm{~d}), 67.1$ (t), $32.5(\mathrm{t}), 29.7(\mathrm{t}), 25.8(\mathrm{q}), 18.1(\mathrm{~s}),-4.4(\mathrm{q}),-4.5(\mathrm{q})$; HRMS Calcd for $\mathrm{C}_{19} \mathrm{H}_{28} \mathrm{O}_{2} \mathrm{Si}\left(\mathrm{M}^{+}\right)$: 316.18586; Found: 316.18665. $4\left(\mathbf{R}_{1}=\right.$ TBS, $\left.\mathbf{R}_{2}=\mathbf{C}_{6} \mathbf{H}_{5}\right)$ : oil; $[\alpha]_{\mathrm{D}}-49^{\circ}$ (c 0.17, $\left.\mathrm{CHCl}_{3}\right) ;{ }^{1} \mathrm{H}$ NMR $\left(400 \mathrm{MHz}, \mathrm{CDCl}_{3}\right): \delta 7.47(\mathrm{~m}, 2 \mathrm{H}), 7.31(\mathrm{~m}$, $3 \mathrm{H}), 4.68(\mathrm{~d}, 1 \mathrm{H}, \mathrm{J}=4.3 \mathrm{~Hz}), 3.94(\mathrm{ddd}, 1 \mathrm{H}, \mathrm{J}=11.6,10.0$, $2.5 \mathrm{~Hz}$ ), 3.85 (ddd, $1 \mathrm{H}, \mathrm{J}=9.0,4.9,4.2 \mathrm{~Hz}$ ), 3.63 (ddd, $1 \mathrm{H}, \mathrm{J}$ $=11.1,4.6,4.2 \mathrm{~Hz}), 1.88(\mathrm{~m}, 1 \mathrm{H}), 1.76(\mathrm{~m}, 2 \mathrm{H}), 1.57(\mathrm{~m}, 1 \mathrm{H})$, $0.90(\mathrm{~s}, 9 \mathrm{H}), 0.11(\mathrm{~s}, 3 \mathrm{H}), 0.10(\mathrm{~s}, 3 \mathrm{H}) ;{ }^{13} \mathrm{C}$ NMR $(100 \mathrm{MHz}$, $\mathrm{CDCl}_{3}$ ): $\delta 131.7$ (d), 128.2 (d), 128.1 (d), 123.1 (s), 87.2 (s), $85.8(\mathrm{~s}), 71.2(\mathrm{~d}), 68.4(\mathrm{~d}), 63.4(\mathrm{t}), 29.5(\mathrm{t}), 29.3(\mathrm{t}), 25.9(\mathrm{q})$, 23.6 (s), - 4.6 (q); HRMS Calcd for $\mathrm{C}_{19} \mathrm{H}_{28} \mathrm{O}_{2} \mathrm{Si}\left(\mathrm{M}^{+}\right)$: 316.18586; Found: 316.18445 . 10: oil; $[\alpha]_{\mathrm{D}}-10.8^{\circ}$ (c 0.63 , $\mathrm{CHCl}_{3}$ ); ${ }^{1} \mathrm{H}$ NMR $\left(400 \mathrm{MHz}, \mathrm{CDCl}_{3}\right): \delta 5.84$ (dddd, $1 \mathrm{H}, \mathrm{J}=$ $17.1,10.2,7.0,6.9 \mathrm{~Hz}$ ), 5.09 (dddd, $1 \mathrm{H}, \mathrm{J}=17.2,3.3,3.3,1.5$ $\mathrm{Hz}$, 5.04 (ddd, $1 \mathrm{H}, \mathrm{J}=10.2,1.0,1.0 \mathrm{~Hz}$ ), 3.95 (dddd, $1 \mathrm{H}, \mathrm{J}=$ $11.3,4.0,2.2,1.9 \mathrm{~Hz}), 3.67($ br s, $1 \mathrm{H}), 3.43(\mathrm{ddd}, 1 \mathrm{H}, \mathrm{J}=$ 11.6, 11.4, 2.4 Hz), 3.32 (ddd, $1 \mathrm{H}, \mathrm{J}=8.1,5.3,1.4 \mathrm{~Hz}$ ), 2.36 (ddd, $1 \mathrm{H}, \mathrm{J}=14.4,8.4,6.8 \mathrm{~Hz}$ ), 2.17 (ddd, $1 \mathrm{H}, \mathrm{J}=14.4,6.8$, $5.6 \mathrm{~Hz}), 1.98(\mathrm{~m}, 1 \mathrm{H}), 1.84(\mathrm{~m}, 1 \mathrm{H}), 1.63(\mathrm{~m}, 1 \mathrm{H}), 1.31(\mathrm{~m}$, $1 \mathrm{H}), 0.92(\mathrm{~s}, 9 \mathrm{H}), 0.66(\mathrm{~s}, 6 \mathrm{H}) ;{ }^{13} \mathrm{C}$ NMR $\left(100 \mathrm{MHz}, \mathrm{CDCl}_{3}\right)$ : $\delta 136.0$ (d), $116.8(\mathrm{t}), 80.2$ (d), $68.1(\mathrm{t}), 67.7$ (d), $36.7(\mathrm{t}), 31.7$ .(t), 26.3(q), 21.0 (t), 18.6(s), - 4.0 (q), - 4.4 (q); HRMS Calcd for $\mathrm{C}_{13} \mathrm{H}_{25} \mathrm{O}_{2} \mathrm{Si}\left(\mathrm{M}^{+}-\mathrm{CH}_{3}\right)$ : 241.16238; Found: 241.16109. 11: oil; $[\alpha]_{\mathrm{D}}+7.3^{\circ}\left(\mathrm{c} 1.54, \mathrm{CHCl}_{3}\right) ;{ }^{1} \mathrm{H}$ NMR $\left(400 \mathrm{MHz}, \mathrm{CDCl}_{3}\right): \delta$ 5.90 (dddd, $1 \mathrm{H}, \mathrm{J}=17.2,10.2,7.2,6.4 \mathrm{~Hz}$ ), 5.10 (ddd, $1 \mathrm{H}, \mathrm{J}=$ $17.2,3.4,1.7 \mathrm{~Hz}$ ), 5.06 (ddd, $1 \mathrm{H}, \mathrm{J}=10.2,2.1,1.2 \mathrm{~Hz}$ ), 3.89 (dddd, $1 \mathrm{H}, \mathrm{J}=11.3,4.0,2.0,1.8 \mathrm{~Hz}$ ), 3.32 (ddd, $1 \mathrm{H}, \mathrm{J}=11.4$, $11.4,4.5 \mathrm{~Hz}$ ), $3.28(\mathrm{~m}, 1 \mathrm{H}), 3.10(\mathrm{ddd}, 1 \mathrm{H}, \mathrm{J}=8.7,8.6,2.8$ $\mathrm{Hz}$ ), 2.58 (ddddd, $1 \mathrm{H}, \mathrm{J}=14.6,6.1,3.0,1.6,1.4 \mathrm{~Hz}$ ), 2.12 (dddd, $1 \mathrm{H}, \mathrm{J}=14.6,7.3,7.3,1.1 \mathrm{~Hz}), 2.02(\mathrm{~m}, 1 \mathrm{H}), 1.64(\mathrm{~m}$, $2 \mathrm{H}), 1.43(\mathrm{~m}, 1 \mathrm{H}), 0.88(\mathrm{~m}, 9 \mathrm{H}), 0.07(\mathrm{~m}, 6 \mathrm{H}) ;{ }^{13} \mathrm{C}$ NMR $(100$ $\left.\mathrm{MHz}, \mathrm{CDCl}_{3}\right): \delta 136.0$ (d), $116.8(\mathrm{t}), 82.7$ (d), 71.3 (d), 68.3 $(\mathrm{t}), 37.0(\mathrm{t}), 34.0(\mathrm{t}), 26.2(\mathrm{q}), 26.1(\mathrm{t}), 18.3(\mathrm{~s}),-3.5(\mathrm{q})$; HRMS Calcd for $\mathrm{C}_{13} \mathrm{H}_{25} \mathrm{O}_{2} \mathrm{Si}\left(\mathrm{M}^{+}-\mathrm{CH}_{3}\right)$ : 241.16238; Found: 241.16584. 